Data from 428 women, were included in this secondary data analysis. All women were asymptomatic for BV at baseline and tested for BV every two months. Persistent cases of $\mathrm{BV}$ were positive for BV at month two, four and six. Women who were negative for BV at month two, four and six were classified as no BV. All other cases were defined as episodic BV. Incident STI was defined as any new case of CT or NG at month eight. Factors associated with STI acquisition were assessed using Binary Logistic Regression. The final model was adjusted for age, education, women who have sex with women and BV status.

Most women were $\leq 21$ years $(55.8 \%)$ and completed some post high school/GED education (50.9\%). There were 179 (41.8\%) women with persistent BV, 204 (47.7\%) women with episodic BV and 45 (10.5\%) women with no BV. At month eight $8.6 \%$ of women tested positive for an STI. Bivariate analysis demonstrated a significant association between women with no BV and STI acquisition $(\mathrm{p}=0.02)$. Women with no BV did not acquire an STI, 7.3\% of women with persistent BV acquired and STI and 11.7\% of women with episodic BV acquired an STI. Women with high school or more than high school education compared to women with less than high school education had decreased odds of developing an STI (Adjusted Odds Ratio (adjOR): 0.38; 95\% CI: 0.15-0.92; and adjOR: 0.31 ; 95\%CI: $0.13-0.73$ ).

At least a high school education was associated with increased odds of STI acquisition compared to less than high school education. STI acquisition was only observed among women with BV.

\section{P418 CORRELATES OF IMMUNE PROTECTION IN THE RABBIT MODEL OF SYPHILIS VACCINATION}

T Reid*, A Haynes, E Romeis, C Godornes, V Campbell, D Koelle, L Giacani. University of Washington, Seattle, USA

\subsection{6/sextrans-2021-sti.443}

Background An effective syphilis vaccine will be integral to efforts to eradicate this disease. Promising vaccine candidates are surface antigens of the syphilis spirochete, Treponema pallidum subsp. pallidum ( $T$. pallidum). These antigens can be targeted by vaccination-induced opsonic antibodies and mediate pathogen immune clearance. Defining correlates of protection will aid in the identification of the best vaccine candidates. Here, we sought to investigate whether immunization with variants of the $\mathrm{T}$. pallidum Repeat $\mathrm{C}$ (TprC) protein and the conserved NH2-terminus of the TprK induced protection and whether splenocyte proliferation and IFN- $\gamma$ production correlated with protection.

Methods Rabbits were immunized with either a cocktail of three recombinant, full-length $\mathrm{TprC}$ variants, or the NH2-terminus of the TprK protein with a RIBI-like adjuvant. Animals were challenged with $\mathrm{T}$. pallidum intradermally (10 sites; $10^{\wedge} 5$ bacteria/site). Treponemal burden and progression to ulceration were monitored. To assess for immunogen-specific splenocytes, pools of synthetic peptides corresponding to each immunogen were used to stimulate splenocytes collected exvivo in proliferation assays. Supernatants from stimulated splenocytes were used to quantify IFN- $\gamma$ responses by ELISA.

Results Immunizations protected animals significantly albeit not completely. At day 35 post-challenge only $14.1 \%$ and $15.5 \%$ of lesions ulcerated in immunized rabbits compared to the
$95 \%$ of lesions in unimmunized rabbits. At day 21, there was a $99.3 \%$ and $98.7 \%$ reduction in treponemal burden averaged across all challenge sites in TprC- and TprK-immunized rabbits compared to unimmunized animals, respectively. Lymphocyte proliferation and IFN- $\gamma$ production correlated to reduction in both percent of ulcerated lesions and treponemal burden.

Conclusions Lymphocyte proliferation and IFN- $\gamma$ release assays may serve as surrogates to assess for antigen-specific T-cell responses. TprK and $\mathrm{TprC}$ immunizations are able to stimulate cellular immunity in a $\mathrm{TH} 2$ environment, which is key to development of an effective syphilis vaccine.

\section{P420 RHEIN INHIBITS CHLAMYDIA TRACHOMATIS INFECTION BY REGULATING PATHOGEN-HOST CELL INTERACTIONS}

X Yu*, W Chen, Q Xu, X Su, Y Xue, H Zheng. Dermatology Hospital, Southern Medical University, Guangzhou, China

\subsection{6/sextrans-2021-sti.444}

Background The global incidence of genital Chlamydia trachomatis (C. trachomatis) infection increased rapidly as the primary available treatment of $\mathrm{C}$. trachomatis infection being the use of antibiotics; however, the development of antibiotics resistant stains and other treatment failures are often observed in patients. Consequently, novel therapeutics are urgently required. Rhein is a monomer derivative of anthraquinone compounds with an anti-infection activity. This study investigated the effects of rhein on treating C. trachomatis infection.

Methods The cytotoxicity of rhein was examined by Cell Counting Kit (CCK) 8 assay. Rhein's inhibitory effect on C. trachomatis infection was detected by immunofluorescent staining, electron microscopy, and progeny infection titer assay. The protein levels of p-RSK, p-ERK, RSK, ERK, cHSP60 were detected by immunoblotting assays. Lastly, C. trachomatis infected mouse model was used to determine the in vivo antiinfection effect of rhein.

Results Rhein showed significant inhibitory effects on the growth of C. trachomatis in multiple serovars of C. trachomatis, including $\mathrm{D}, \mathrm{E}, \mathrm{F}$, and $\mathrm{L} 1$, and in various host cells, including HeLa, McCoy, and Vero. Rhein could not directly inactivate C. trachomatis but could inhibit the growth of C. trachomatis by regulating pathogen-host cell interactions. Host p-ERK and p-RSK were both down-regulated in the presence of rhein at $36 \mathrm{~h}$ and $48 \mathrm{~h}$ post-infection. Combined with azithromycin, the inhibitory effect of rehin was synergistic both in vitro and in vivo.

Conclusion We found rhein inhibited C. trachomatis infection regulating pathogen-host cell interactions. Together these findings suggest that rhein could be developed for the treatment of C. trachomatis infections.

\section{P423 TAKING REAL ISSUES TO VIRTUAL CONFERENCES: THE TRAINING OF YKP TO DISCUSS SOCIAL VULNERABILITY MEASURES IN THE COVID-19 CONTEXT}

D Agostinho Calixto*, N Fagundes Correia, C Moreira Aló, C Bernardes Sousa. Ministry of Health of Brazil

10.1136/sextrans-2021-sti.445 